\title{
Usefulness of Mobile Devices in the Diagnosis and Rehabilitation of Patients with Dizziness and Balance Disorders: A State of the Art Review
}

This article was published in the following Dove Press journal: Clinical Interventions in Aging

\author{
Anna Gawronska (D) \\ Anna Pajor ${ }^{2}$ \\ Ewa Zamyslowska-Szmytke ${ }^{3}$ \\ Oskar Rosiak (D) \\ Magdalena Jozefowicz- \\ Korczynska $\mathbb{1}^{\prime}$
}

'Balance Disorders Unit, Department of Otolaryngology, Medical University of Lodz, The Norbert Barlicki Memorial Teaching Hospital, Lodz, Poland; ${ }^{2}$ Department of Otolaryngology, Head and Neck Oncology, Medical University of Lodz, The Norbert Barlicki Memorial Teaching Hospital, Lodz, Poland; ${ }^{3}$ Balance Disorders Unit, Department of Audiology and Phoniatrics, Nofer Institute of Occupational Medicine, Lodz, Poland
Correspondence: Anna Gawronska Balance Disorders Unit, Department of Otolaryngology, Medical University of Lodz, The Norbert Barlicki Memorial Teaching Hospital, Kopcinskiego 22, Lodz 90-I53, Poland

Tel +48663742373

Email gawronska90@gmail.com
Objective: The gold standard for objective body posture examination is posturography. Body movements are detected through the use of force platforms that assess static and dynamic balance (conventional posturography). In recent years, new technologies like wearable sensors (mobile posturography) have been applied during complex dynamic activities to diagnose and rehabilitate balance disorders. They are used in healthy people, especially in the aging population, for detecting falls in the older adults, in the rehabilitation of different neurological, osteoarticular, and muscular system diseases, and in vestibular disorders. Mobile devices are portable, lightweight, and less expensive than conventional posturography. The vibrotactile system can consist of an accelerometer (linear acceleration measurement), gyroscopes (angular acceleration measurement), and magnetometers (heading measurement, relative to the Earth's magnetic field). The sensors may be mounted to the trunk (most often in the lumbar region of the spine, and the pelvis), wrists, arms, sternum, feet, or shins. Some static and dynamic clinical tests have been performed with the use of wearable sensors. Smartphones are widely used as a mobile computing platform and to evaluate the results or monitor the patient during the movement and rehabilitation. There are various mobile applications for smartphone-based balance systems. Future research should focus on validating the sensitivity and reliability of mobile device measurements compared to conventional posturography.

Conclusion: Smartphone based mobile devices are limited to one sensor lumbar level posturography and offer basic clinical evaluation. Single or multi sensor mobile posturography is available from different manufacturers and offers single to multi-level measurements, providing more data and in some instances even performing sophisticated clinical balance tests.

Keywords: mobile posturography, dizziness, imbalance, clinical tests with sensors, telephone applications, older adults

\section{Introduction}

The aging of the balance system presents as dizziness, disequilibrium, and vertigo. It causes stress and isolation, and it results in a reduction of physical activities in the older adults due to an increased risk of falling. Community surveys show dizziness affects more than $30 \%$ of people over 70 and it is one of the most commonly reported medical complaints, affecting $15-35 \%$ of the adult population. ${ }^{1}$ Saber Tehrani et $\mathrm{al}^{2}$ stated that in 2011, there were an estimated 3.9 million visits to U. $\mathrm{S}$ emergency departments for complaints of dizziness or vertigo. In a review of 16 studies on the economic evaluation of vertigo, up to 9.6 visits were reported 
annually at the primary care provider, up to 7.2 visits to a specialist, and there were up to 6 instrumental diagnostic procedures. ${ }^{3}$ Epidemiological data indicate a higher prevalence among women $(27 \%)$ than men $(14 \%)$, and an increase in prevalence with age. ${ }^{4}$ In a US study, the national costs for patients presenting with dizziness to emergency departments were estimated to exceed $\$ 4$ billion per year. Of those, $25.7 \%$ were of otologic/ vestibular origin. ${ }^{2}$

For many years, posturographic examination was considered the golden standard for objective body posture examination. It is based on an inverted pendulum model and the Center of Pressure (COP) is assumed to be the free end of the pendulum. Tracking the displacement of the COP projection in a two-dimensional plane is done by registering the pressure of the feet on a posturographic platform, which is read by detectors, converted into a digital signal, and transmitted to a computer. There is no international consensus as to which of the parameters describing the displacement of COP have significant clinical value in the diagnosis of balance disorders. ${ }^{5}$ The literature emphasizes the value of the Center of Mass (COM) test and determining the relationship of COM to COP. There is also doubt regarding the significance of the COP evaluation on a posturographic platform in static conditions. A further development of this research method is computerized dynamic posturography, in which the researcher can force ground motions and change the visual reference points, thereby enabling the clinician to assess the patient's compensatory mechanisms by analysing COP displacements. This type of posturography allows for a more functional assessment of the range of postural stability as well as the dependence of the equilibrium system on information from the vision organ.

In recent years, new technologies have been applied in many disciplines for health monitoring, especially in physical activity assessment, specifically miniaturized wearable, body-fixed motion devices such as switches, pedometers, actometers, goniometers, accelerometers, and gyroscopes. ${ }^{6}$ These new inventions contributed to the development of mobile posturography, which enables COM testing during complex dynamic activities - both everyday life tasks and in clinical/laboratory conditions. Mobile posturography can also be utilized in assessing balance disorders and patient rehabilitation. ${ }^{7}$ It is especially aimed at older patients, as the aging of the world's population and the increasing number of active old people demand new non-invasive methods to evaluate balance and gait disorders. New wearable devices could predict falls and make it possible to introduce training programs tailored to older adults. It could not only improve the wellbeing of the aging population, but it could also have additional economic and social benefits.

The aim of this state of the art review is to present the usefulness of various mobile devices as cutting edge technology which could replace conventional posturography in the diagnosis and rehabilitation of dizziness and balance instability. We focused only on devices which had at least trunk worn sensor, as the trunk is proposed to be the most optimal region for single sensor mobile posturography. We also analysed the possibility of performing static and dynamic clinical tests with the use of mobile devices.

\section{Types of Mobile Devices}

Various types of mobile devices have been described in balance disorders diagnosis and treatment. We have summarized the information on mobile devices, with at least one trunk worn sensor, available from literature in Table 1. A commercially available device, the VertiGuard $^{\circledR}$ D/RT system (VestiCure GmbH, Germany, now Otocure GmbH, Metzingen, Germany), was frequently used. It is a waistworn vibrotactile system that consists of two gyrometers and a battery-driven main unit fixed on a belt at the COM. It records the angular acceleration of the patient's body in the sagittal and horizontal planes during the patient's daily tasks. ${ }^{8}$ The system is available in a diagnostic (D) version and a more complex therapeutic (RT) version, which has additional vibrators on the front, back, left, and right sides that can induce vibrotactile feedback signals. ${ }^{9}$ The VertiGuard ${ }^{\circledR}$ device was used in balance rehabilitation in the older adults population and to assess body sway in patients with chronic uncompensated vestibular disorders. ${ }^{7,10}$ Its use was further extended to balance training with neurofeedback in patients with different balance disorders (like canal paresis, otolith disorder, peripheral vestibular impairment after removal of an acoustic neuroma, microvascular compression syndrome, Parkinson's disease (PD), or presbyvertigo) and in identifying fallers in PD. ${ }^{9-11}$

The SwayStar ${ }^{\mathrm{TM}}$ system (Balance International Innovations $\mathrm{GmbH}$, Iseltwald, Switzerland, and BESTecetp $\mathrm{GmbH}$, Germany) is another commercially available mobile device, which is mounted on a belt at the back. It has two angular velocity transducers oriented in roll and pitch planes, and it directly measures the angular deviations of the trunk near the COM (around L3-L5). ${ }^{12,13}$ It 
Table I Type of Mobile Devices

\begin{tabular}{|c|c|c|c|c|c|}
\hline Location & Name & Placement & Sensors & Main Application & References \\
\hline \multirow[t]{3}{*}{$\begin{array}{l}\text { One level } \\
\text { sensors }\end{array}$} & $\begin{array}{l}\text { Dynaport MiniMod } \\
\text { Hybrid }^{\circledR}\end{array}$ & Lower back & $\begin{array}{l}\text { ACC } \\
\text { GYR }\end{array}$ & $\begin{array}{l}\text { Balance diagnostics gait analysis } \\
\text { identification of fallers }\end{array}$ & {$[17,20-22]$} \\
\hline & $\begin{array}{l}\text { VertiGuard }^{\circledR} \mathrm{D} / \mathrm{RT} \\
\text { system }\end{array}$ & Lower back & GYR & $\begin{array}{l}\text { Rehabilitation balance diagnostics } \\
\text { identification of fallers }\end{array}$ & {$[7-11]$} \\
\hline & BalanceBelt & Lower back & $\begin{array}{l}\text { ACC } \\
\text { GYR }\end{array}$ & Rehabilitation & [32] \\
\hline \multirow[t]{5}{*}{$\begin{array}{l}\text { Multi-levels } \\
\text { sensors }\end{array}$} & Movit $^{\circledR}$ sensor & $\begin{array}{l}\text { Obligatory: lower back } \\
\text { Optional: thighs, shanks, feet }\end{array}$ & $\begin{array}{l}\text { ACC } \\
\text { GYR }\end{array}$ & Balance diagnostics gait analysis & [33] \\
\hline & $\begin{array}{l}\text { The SwayStar }{ }^{\mathrm{TM}} \\
\text { system }\end{array}$ & $\begin{array}{l}\text { Obligatory: lower back } \\
\text { Optional: head }\end{array}$ & GYR & Balance diagnostics & {$[12-14]$} \\
\hline & $\begin{array}{l}\text { APDM's Opal- } \\
\text { Mobility Lab }{ }^{\mathrm{TM}}\end{array}$ & $\begin{array}{l}\text { Lower back, wrists, arms, } \\
\text { sternum, legs, feet }\end{array}$ & $\begin{array}{l}\text { ACC } \\
\text { GYR } \\
\text { MAG }\end{array}$ & $\begin{array}{l}\text { Balance diagnostics gait analysis balance } \\
\text { monitoring }\end{array}$ & [15-19] \\
\hline & Xsens device & $\begin{array}{l}\text { Lower back, head, trunk, wrists, } \\
\text { arms, legs, feet }\end{array}$ & $\begin{array}{l}\text { ACC } \\
\text { GYR }\end{array}$ & Balance diagnostics & {$[23,24]$} \\
\hline & Physilog $^{\circledR}$ & $\begin{array}{l}\text { Lower back, trunk, wrists, legs, } \\
\text { feet }\end{array}$ & $\begin{array}{l}\text { ACC } \\
\text { GYR } \\
\text { MAG }\end{array}$ & Gait analysis & [25] \\
\hline
\end{tabular}

Abbreviations: ACC, accelerometer; GYR, gyroscope; MAG, magnetometer.

can be equipped with an additional vibrotactile and auditory biofeedback unit - Balance Freedom ${ }^{\mathrm{TM}}$ - in which signal transducers are mounted on a head-band. ${ }^{12}$ FaraldoGarcia et $\mathrm{al}^{14}$ established population age-adjusted reference values for the Sway Star system. The test results can be summarized with an option called balance control summary, which is an analysis of 14 balance tests.

Some researchers have used Opal, another commercial wearable system with inertial sensors, with the associated Mobility Lab ${ }^{\mathrm{TM}}$ Software (APDM, Inc. Portland, OR, USA, https://apdm.com) ${ }^{15-19}$ The system consists of up to six sensors, each containing 3-axis accelerometers, 3-axis gyroscopes, and 3-axis magnetometers. The sensors are attached to the wrists, arms, sternum, lumbar region, feet, and shins. Clinical tests, like the instrumented Timed Up and Go, Two-minute Walk, and Postural Sway tests can be performed using Mobility $\mathrm{Lab}^{\mathrm{TM}}{ }^{16}$ Sankarpandi et $\mathrm{al}^{18}$ demonstrated good to excellent test-retest reliability of the instrumented tests (iTUG, iSway) using the Opal-Mobility $\mathrm{Lab}^{\mathrm{TM}}$ system in 27 patients with uni/bilateral vestibular loss. The system was validated for gait analysis in young children. Compared with 3D motion capture, the temporal parameters were similar between the two systems, but the spatial ones may be used with a correction. ${ }^{19}$ The Mobility $\mathrm{Lab}^{\mathrm{TM}}$ system was also used in monitoring balance and gait in $\mathrm{PD} .{ }^{16,17}$

A few authors have used Dynaport MiniMod Hybrid ${ }^{\circledR}$ (McRoberts BV, The Hague, The Netherlands, https:// www.mcroberts.nl/). This device is fixed on a belt at the lower back (at the level of L4-5), and it consists of a triaxial accelerometer and a tri-axial gyroscope. ${ }^{17,20}$ It was tested in older adults to assess the strategies used to counteract imbalance during unsupported standing and to evaluate mobility performance and its correlation with Parkinsonian gait. ${ }^{20,21}$ Iluz et $\mathrm{al}^{22}$ recorded data in 40 patients with PD using Dynaport, and they developed an algorithm for objective and automated detection of missteps in these patients' daily lives, which may contribute to the early identification of fallers.

Another solution used in research is the Xsens device (Xsens, Enschede, The Netherlands www.xsens.com), which is composed of six body-worn sensors, each consisting of a 3-dimensional gyroscope and a tri-axial accelerometer. ${ }^{23}$ This device was used in a study by Spain et $\mathrm{al}^{24}$ who recorded balance in MS patients with normal walking speed compared to healthy controls. They 
observed that body-worn sensors can detect early mobility changes in contrast to stopwatch timed tests. Physilog ${ }^{\circledR}$ (GaitUp, Lausanne, Switzerland https://gaitup.com/), an inertial measurement unit (IMU) sensor in its latest version as Physilog ${ }^{\circledR} 6$, is a $10 \mathrm{D}$ sensor that includes a high-quality 3D accelerometer, 3D gyroscope, 3D magnetometer, and a barometric pressure sensor. The manufacturer developed additional research software, the PhysiGait Lab, specifically for gait analysis (assessing 26 parameters) in movement science and neurology (PD, stroke, SM). These sensors are attached to each wrist, one on each shank, and one on the trunk, complemented with one device on each foot and one on the back (lumbar 3); they were used in post-stroke patients to verify the validity of the iTUG test. $^{25}$

In balance diagnostics and rehabilitation, many different prototype mobile devices have been developed. ${ }^{26-31}$ Kingma et $\mathrm{al}^{32}$ proposed the BalanceBelt (Elitac Wearables, Utrecht, The Netherlands) worn around the waist. It is a vibrotactile system with sensors (6 DOFaccelerometer and gyroscope) fixed at the back and 12 tactors positioned in a belt equally distributed around the belt. An inclination of more than 2.5 degrees in any direction will activate a tactor, sending a vibration to the patient. The system was tested on patients with severe bilateral vestibular loss, and a significant improvement in daily life was observed in 23 out of 39 patients. Alessandrini et $\mathrm{al}^{33}$ used a Movit ${ }^{\circledR}$ sensor (Captiks Srl, Rome, Italy; http://www.captiks.com/) with 3D accelerometers mounted on the posterior trunk near the COM. Movit System G1 is a wearable wireless system for motion capture and analysis. The authors confirmed the correlation between the parameters measured by mobile devices and forceplate-based posturography in patients with unilateral vestibular failure and in healthy subjects. ${ }^{33}$

\section{Types and Location of Sensors}

Wearable sensors used for body motion assessment may be categorized as inertial motion sensors and plantar force sensors. ${ }^{34}$ Many devices use accelerometers (linear acceleration measurement), gyroscopes (angular acceleration measurement), and magnetometers (heading measurement relative to the Earth's magnetic field). ${ }^{35}$ They are often based on microelectromechanical systems (MEMS), which enabled the development of miniaturized inertial sensors a key technology in the process of making wearable devices smaller, lighter, and more comfortable. ${ }^{36}$ In a review of 26 balance studies in PD, $69 \%$ of researchers used triaxial accelerometers, $27 \%$ - inertial sensors, and $4 \%$ - other types of sensors (1D and 2D gyroscopes). ${ }^{37}$

Similar data were reported by other authors; thus, the accelerometer was the most often used sensor $(70 \%)$ in the group of single-sensor devices. ${ }^{38}$ It was used as the only inertial sensor in $70 \%$ of the 40 studies reviewed by Howcroft et $\mathrm{al}^{39}$ for fall risk assessment in people aged 60 and over, while accelerometers and gyroscopes were used in $27.5 \%$ of studies. Rucco et $\mathrm{al}^{38}$ pointed out that some features of the accelerometer, such as its low cost, small size and weight, long-lasting batteries, and the number of commercially available solutions, have contributed to it being used the most frequently in mobile devices.

A tendency to reduce the number of sensors in mobile devices has been observed, so the devices can be more easily adopted by older patients. Regarding the number of sensors, in a review of 42 articles on the type and location of wearable sensors in healthy aged people, one sensor was used in 16 papers, two sensors were used in 17 papers, and three or more simultaneous sensors were used in only nine papers. ${ }^{38}$

Sensors in mobile devices are mounted on various parts of the body, but most often on the trunk (the lumbar region of the spine or the pelvis), and less often on the lower limbs, the head, upper limbs, and the chest. The lower back was the only location of sensors in $60-65 \%$ of studies. $^{38,39}$ However, in $30 \%$, sensors were placed on the feet (as pressure sensors within shoes), using two or three sensors. ${ }^{38}$

In a study by Özdemir, ${ }^{40} 14$ healthy young volunteers wearing six sensors attached to different body parts participated in 16 types of daily activities and 20 types of falls (so, the dataset consists of 2520 movements). It was demonstrated that the waist is the best region for a single sensor location on the body for wearable fall detection systems, with an average $98.42 \%$ accuracy by six machine learning techniques. It was followed by a thigh sensor (97.89\% accuracy), ankle sensor (97\% accuracy), head sensor (96.61\% accuracy), and chest sensor $(96.5 \%$ accuracy). The least accurate was the wrist-mounted sensor (94.92\% accuracy). The trunk is considered the optimal place for the location of the sensors because, in this position, the sensor is the closest to the COM. ${ }^{41}$ There are some doubts as to the position of sensors on the head because although the vestibular system is located in this region, the accuracy of the sensors was lower than for the trunk. ${ }^{40}$ However, in older people, the head-based model 
was better at classifying fallers, and multi-sensor models were better at classifying non-fallers. ${ }^{42}$

\section{Instrumented Static and Dynamic Clinical Tests}

Clinicians are constantly looking for objective methods to quantitively evaluate balance deficit for diagnosis and therapy. Computerized systems such as posturography and wearable inertial sensors could provide more sensitive, specific, and repetitive testing in clinical practice. A serious disadvantage of such complex systems, such as CDP and gait laboratories, is that they are very expensive, and they require a large room and trained staff to perform the tests, which limits their availability to tertiary reference centres.

The Romberg test is one of the most commonly used clinical bedside examinations of postural stability. The iSway is an instrumented Romberg test obtained from wearable signals (accelerometers or gyroscopes) placed on, eg, the trunk, which measure the patient's sway. It provides useful quantitative information on postural balance, which can substitute clinical balance scales. It is used for screening for balance problems and vestibular disorders. The most frequent variation is the double leg stance for $30 \mathrm{~s}$ with eyes open and closed, although some authors also include different surface conditions, such as standing on a firm and foam surface, or a different stance, eg, tandem, semi-tandem, or single-leg stance. There is evidence that the results of sway tests from iSway using body-worn accelerometers (ACC) are useful and comparable with posturographic evaluation. Mancini et $\mathrm{al}^{43}$ performed tests on subjects with early PD and on controls, in which they measured and compared the postural sway from the force plate and iSway using a body-worn ACC. They suggest that iSway measurements are more sensitive, reliable, and valid for characterizing posture control in PD subjects. Sankarpandi et $\mathrm{al}^{18}$ recorded iSway results (Mobility Lab ${ }^{\mathrm{TM}}$ ) for 27 patients with vestibular deficit and concluded that this wearable system identified and distinguished fallers from non-fallers.

Another standing balance test performed with wearable inertial sensors is the balance error scoring system (BESS). The standard protocol includes three different stances: feet together, one foot, and tandem stance on two different types of surface - firm and foam (six conditions in total). A modified, shorter version of the BESS (mBESS) includes three different stances but limited only to a firm surface. In the BESS test, the examiner notes the failures according to the standardized balance errors list, in each 20 -second trial, and scores them appropriately. ${ }^{44,45}$

Brown et $\mathrm{al}^{44}$ validated an algorithm to objectively measure BESS using a wireless inertial sensor on the forehead in healthy subjects. Their preliminary results suggest that an instrumented mBESS test may provide enough balance information to predict total BESS scores. King et $\mathrm{al}^{45}$ also analysed the BESS test in adolescent athletes after mild traumatic brain injury, using an inertial sensor (Opal by APDM, Inc) placed at L-5 with an elastic belt. The mBESS results had the highest diagnostic accuracy for distinguishing post-traumatic patients from healthy individuals. The National Institutes of Health (NIH) Balance Toolbox suggests using inertial sensors to perform the Standing Balance Test for general balance screening (the measurement of anteroposterior postural sway). $^{45}$

A different, complex balance test is the Clinical Test of Sensory Integration for Balance (iCTSIB), which in some ways resembles the sensory organization test (SOT) in CDP. Patients stand on a firm surface or on foam, with eyes open or closed, to quantitively assess the use of surface, visual, and vestibular information for postural sway control. Whitney et $\mathrm{al}^{46}$ examined healthy adults with a wide age range to find a relationship between ACC measured at the pelvis and the center of pressure obtained from CDP (EquiTest ${ }^{\circledR}$ ) in a SOT test. The subjects completed three consecutive trials for each of the six SOT conditions. They found an association between COP and ACC measurements, even when using the first trial. The iCTSIB was recommended as a screening test for sensory system performance in neurological patients.

One of the most useful clinical tests to evaluate both static and dynamic balance and mobility is the Timed Up and Go test (TUG). Measured with a stopwatch, it uses the time that a person takes to rise from a chair, walk three meters, turn around, walk back to the chair, and sit down. However, a limitation of TUG is that it focuses only on the total time, without focusing on different subphases of the subject's movements. Wearable inertial sensors could be used to record and analyse each of the gait subcomponents (walking, turning, rising from the chair, and sitting down) of the TUG test. Salarian et $\mathrm{al}^{47}$ compared the results of a conventional TUG with stopwatch measurements and the iTUG, which employed seven portable inertial sensors in 20 subjects in the early stages of PD and in a control group. The authors concluded that iTUG showed 
significant differences between early PD and healthy individuals in the number of steps per minute, the angular velocity of arm-swing, turning duration, and the time to perform turn-to-sits. In their opinion, out of all the iTUG subcomponents, the gait, turning, and turn-to-sit were most sensitive. Similarly, Zampieri et $\mathrm{al}^{48}$ showed that turning might be the most challenging movement for PD patients, and that the turning phase in TUG seems to be the most sensitive for detecting impairments. Gerhardy et $\mathrm{al}^{49}$ used iCTSIB with inertial sensors and correlated these results with the smartphone-based version of the iTUG. One of their conclusions was that the performance of the vestibular and somatosensory system is strongly correlated with iTUG total time.

The utility of wearable systems in distinguishing fallers from non-fallers was confirmed by Sankarpandi et $\mathrm{al}^{18}$ who recorded the results of the iTUG and iSway in two sessions in three consecutive measurements, and they confirmed good to excellent reliability of both systems' parameters. O'Sullivan et $\mathrm{al}^{50}$ also stated that an accelerometer could distinguish between fallers and non-fallers, show different balance conditions, and possibly correlate with clinical tools such as the Berg Balance Scale (BBS) and TUG.

Other applications of mobile devices included mobility tests like the Stand and Walk (ISAW) test and the Long Walk plugin (IWalk). Patients wear a Mobility Lab ${ }^{\text {TM }}$ system, which makes it possible to calculate gait variability, phase coordination index, turning, and asymmetry. ${ }^{15}$ This kinematic data can be used to quantitively measure progress in rehabilitation. The vast amount of data and parameters measured by mobile devices and their sensors required the employment of machine-learning techniques to develop algorithms capable of interpreting these datasets. The authors mentioned above recommend using wearable sensors to perform iTUG, Sway (iSway), Stand and Walk (ISAW), and Long Walk plugin (IWalk) tests. ${ }^{15,18,47-50}$ The instrumented tests allow for an objective, low-cost quantification of balance performance, and they are more sensitive than clinical examinations.

\section{Patient Notification and Telephone Applications (Apps)}

Mobile phone technology has had a major impact on the development of monitoring systems that are based on wearable sensors. ${ }^{36}$ Smartphones (SP) are widely-used electronic devices that comprise several sensors like an accelerometer, magnetometer, gyroscope, global positioning system, microphone, and camera. SP operating systems are capable of multitasking and can run processes in the background, therefore allowing them to function as a mobile computing platform, which could be used in fall detection and prevention. ${ }^{51}$

Rey-Martinez et $\mathrm{al}^{52}$ created the RombergLab application for the iPhone's operating system, which consists of full posturography software based on a wireless IMU. Lee et al ${ }^{53}$ designed, developed, and assessed a smartphone-based vibrotactile feedback system for balance rehabilitation training at home. The tool included an iPhone, with its tri-axial linear accelerometer, and a "tactor bud" plugged into the SP's audio jack. This instrument was used to measure body acceleration, and anterior-posterior and medial-lateral body tilt. Using variations of Romberg test (with eyes open/closed, semi-tandem, and tandem), healthy subjects and those with vestibular deficits corrected their posture in response to vibration feedback.

The smartphone balance training system could be used at home without difficulty. Another smartphone-based system for balance training and rehabilitation in older people was proposed by Fleury et al. ${ }^{54}$ An application called iBalance-ABF sends audio feedback to the user through an earphone when the tilt angle of the trunk exceeds an adjustable and predetermined threshold. The audio assistance allowed the patient to decrease trunk sway in the tandem stance with closed eyes and to maintain this destabilizing position.

Mellone et $\mathrm{al}^{51}$ presented the results of the FARSEEEING group (an EU consortium to develop fallrelated technologies, databases, and telemedical service model) regarding research on using SPs for fall detection and fall prevention. For fall detection, the uFall application was developed, where real-time fall-detection algorithms already proposed in the literature have been used. The app continuously records the signals from inertial sensors and runs another process that simultaneously acts as a fall/event detector. When a fall is suspected, an audio alarm is generated, and the user has to manually disable it. The uFall app is configured by a clinician before the patient uses it for the first time, and then, during the motor activities, its use is completely transparent to the user. There is still no validation on the uFall fall-detection algorithm. Another app for fall detection is the iFall, which continuously records data from the SP's accelerometer. ${ }^{55}$ The user has to respond to the app when a fall is suspected, otherwise the system triggers an alarm. 
The first attempt to instrument a clinical functional test was called uTUG. ${ }^{51}$ The uTUG is performed with an SP on the lower back. The app records and processes the signals from the accelerometer and gyroscope and then sends a report to a remote server. The uTUG proved to be a pocket-sized tool for fast screening, assessment, and follow-up.

Cohen et $\mathrm{al}^{56}$ used an inertial motion Bluetoothenabled IMU (iPod, Apple, Inc.) unit to measure kinematic variables in a version of the Romberg test with the head still or moving in healthy controls and in patients with benign paroxysmal positional vertigo, postoperative acoustic neuroma resection, and chronic peripheral unilateral weakness. The subjects were instructed to move their heads in time with a specific type of tone.

Only Romberg tests on foam with eyes closed, with the head still or moving, were useful in screening for vestibular impairments, especially in older people. Another application for monitoring human mobility and falls is the Mover. ${ }^{57}$ This app categorizes activities as "Sleeper, Sitter, Lagger, Walker, Mover, Hyper." It also employs an experimental fall-detection algorithm. When a fall is suspected, the Mover will play an alert, which will notify an emergency contact unless disarmed.

\section{The Use of Mobile Devices in the Assessment and Rehabilitation of Balance Disorders}

Mobile devices make it possible to assess posture and gait in both clinical tests and everyday life activities. They are portable, lightweight, and less expensive than classic balance assessment systems (static and dynamic posturography). For these reasons, there is increased interest in their use in diagnostics and rehabilitation. ${ }^{37,58}$ They were used to evaluate posture and gait disorders in healthy people, especially in the aging population, and to detect falls in the older adults. ${ }^{59-61}$ They were employed in the rehabilitation of various neurological diseases, like PD, SM, Alzheimer's disease, and stroke, but also in osteoarticular and muscular system diseases, in diabetes mellitus, and in vestibular disorders. ${ }^{18,24,27,37,62,63}$

In PD, the use of various types of mobile devices to assess posture and gait has been studied to differentiate patients at high risk of falls, different types of disease, carriers of the LRRK2 gene, and people at high risk of developing this disease. ${ }^{37,62,64-66}$ Rovini et al ${ }^{67}$ identified five major applications for wearable devices in PD: early diagnosis, tremor detection, analysis of motor performance, analysis of motor fluctuations (on/off phases), and home and long-term patient monitoring. Some authors reported that postural instability and some features of gait analysis, such as jerk, harmonic stability, oscillation range measured by accelerometers on the pelvis and head, and asymmetry between the right and left side, could be biomarkers for the prodromal phase of $\mathrm{PD} .{ }^{67}$ Hasegawa et al ${ }^{68}$ reported that in PD patients, the most sensitive domains of balance dysfunction were anticipatory postural adjustments prior to gait initiation, and dynamic balance while walking (gait); those measures were significantly correlated with the severity of the disease.

Mobile devices have also been used in rehabilitation and balance training with biofeedback. ${ }^{34}$ Brugnera et al ${ }^{69}$ observed that that vibro-tactile stimulation from the Vertiguard $^{\mathrm{TM}}$ device improved the body balance of patients who did not respond well to conventional vestibular rehabilitation. In PD, a significantly higher increase in the SOT score was reported in patients using the VertiGuard ${ }^{\circledR}$-RT vibrotactile neurofeedback system in comparison to CPD training. ${ }^{9}$

Numerous studies have attempted to establish criteria (eg, the location of sensors, the most useful variables to assess fall risk, classification models) to identify people at high risk of falling (fallers/non-fallers) using wearable sensors. ${ }^{18,36,39,62,70}$ This problem was researched by an international team in the ProFaNE (Prevention of Falls Network Europe) network, which was part of a broader project, "STate of the Art Robot-Supported assessments," or STARS; however, no consensus regarding parameters that are most useful to objectively assess the risk of falls was established. ${ }^{71}$

\section{Limitations}

Limitations on the use of mobile devices in clinical practice result from the fact that many studies describe prototype devices with different locations and number of sensors, and no consensus has been reached regarding the test protocols or derived parameters. ${ }^{68}$ The methodologically poor quality of research is emphasized. There is a lack of standardization in data acquisition, mathematical models, and algorithms used to process data. Hubble et al ${ }^{37}$ in a review of 26 papers on the use of mobile systems in PD only three papers were characterized by research of high methodological quality. Alessandrini et $\mathrm{al}^{33}$ also underline the importance of validation, sensitivity, and the reliability of mobile device measurements compared 
to conventional posturography. In healthy subjects, Faraldo-Garcia et $\mathrm{al}^{59}$ reported that results of computed dynamic posturography and the SwayStar system were significantly related only when the measurements were performed simultaneously and under the same sensory stimulation conditions. Future research should focus on expanding the studied populations and selecting more appropriate patients. Research protocols lack information on patients' assessment for vision, sensation, neuro-motor skills, cognitive functions, and other systemic diseases that may affect the diagnosis and rehabilitation of balance disorders. ${ }^{72}$ It is especially important in older patients, in whom balance disorders are caused by ultrastructure damage, such as the loss of sensory cells, otoconia, and vestibular neurons. ${ }^{73}$ These lesions change the postural strategy and cause greater dependence on visual and proprioceptive signals in the older adults.

\section{Conclusions}

Smartphone based mobile posturography applications are easy-to-use and affordable solutions, but are limited to one sensor lumbar level posturography and offer only basic clinical evaluation (TUG) and limited biofeedback capabilities. Therefore, more research is needed to develop solutions to provide continuous monitoring, improve biofeedback necessary to implement these solutions into home rehabilitation regimens and to decrease the number of false alarms.

Single or multi sensor, commercially available mobile posturography devices offer multi-level measurements, which seem to provide more possibilities for balance monitoring and testing in clinical settings. However the cost and size of these devices makes them impractical to use at home, therefore significantly limiting their capability for continuous monitoring and home rehabilitation.

\section{Abbreviations}

COP, center of pressure; COM, center of mass; CDP, computerized dynamic posturography; IMU, inertial measurement unit; SP, smartphone; PD, Parkinson disease; MS, multiple sclerosis; BESS, balance error scoring system; iCTSIB, Clinical Test of Sensory Integration for Balance; SOT, sensory organization test; TUG, Timed Up and Go test; BBS, Berg Balance Scale; app, smartphone application.

\section{Acknowledgments}

This research was funded by NCBiR, STRATEGMED 2/ 266299/19NCBR/201.

\section{Disclosure}

The authors report no conflicts of interest in this work.

\section{References}

1. Jahn K. The aging vestibular system: dizziness and imbalance in the elderly. Adv Otorhinolaryngol. 2019;82:143-149. doi:10.1159/ 000490283

2. Saber Tehrani AS, Coughlan D, Hsieh YH, et al. Rising annual costs of dizziness presentations to U.S. emergency departments. Acad Emerg Med off J Soc Acad Emerg Med. 2013;20(7):689-696. doi:10.1111/acem. 12168

3. Kovacs E, Wang X, Grill E. Economic burden of vertigo: a systematic review. Health Econ Rev. 2019;9(1):37. doi:10.1186/ s13561-019-0258-2

4. Neuhauser HK. The epidemiology of dizziness and vertigo. Handb Clin Neurol. 2016;137:67-82. doi:10.1016/B978-0-444-634375.00005-4

5. Kingma H, Gauchard GC, De Waele C, et al. Stocktaking on the development of posturography for clinical use. J Vestib Res. 2011;21 (3):117-125. doi:10.3233/VES-2011-0397

6. Yang CC, Hsu YL. A review of accelerometry-based wearable motion detectors for physical activity monitoring. Sensors. 2010;10 (8):7772-7788. doi:10.3390/s100807772

7. Basta D, Rossi-Izquierdo M, Soto-Varela A, Ernst A. Mobile posturography: posturographic analysis of daily-life mobility. Otol Neurotol. 2013;34(2):288-297. doi:10.1097/MAO.0b013e31 $8277 \mathrm{a} 29 \mathrm{~b}$

8. VertiGuard $^{\circledR}$ D/RT. Diagnostic (D) and therapeutic (RT) device for vestibular and stance/gait disorders User's Manual.

9. Rossi-Izquierdo M, Ernst A, Soto-Varela A, et al. Vibrotactile neurofeedback balance training in patients with Parkinson's disease: reducing the number of falls. Gait Posture. 2013;37(2):195-200. doi:10.1016/j.gaitpost.2012.07.002

10. Basta D, Rossi-Izquierdo M, Soto-Varela A, et al. Efficacy of a vibrotactile neurofeedback training in stance and gait conditions for the treatment of balance deficits: a double-blind, placebo-controlled multicenter study. Otol Neurotol. 2011;32 (9):1492-1499. doi:10.1097/MAO.0b013e31823827ec

11. Rossi Izquierdo M, Basta D, Rubio-Rodríguez JP, et al. Is posturography able to identify fallers in patients with Parkinson's disease? Gait Posture. 2014;40(1):53-57. doi:10.1016/j.gaitpost.2014.02.003

12. SwayStar TM. Soft Manual (JHJ Allum, F Honegger) Version 2.1 in $\mathrm{BF}$ File 4.3.7.279. Balance International Innovations $\mathrm{GmbH}$ [homepage on the Internet]; Available from https://www.b2i.info/download/ SwayStarUK.pdf. Accessed September 08, 2020.

13. Rossi Izquierdo M, Santos-Pérez S, Del-Rio Valeiras M, et al. Is there a relationship between objective and subjective assessment of balance in elderly patients with instability? Eur Arch Otorhinolaryngol. 2015;272(9):2201-2206. doi:10.1007/s00405-014-3122-3

14. Faraldo-Garcia A, Sandos-Perez S, Labella - Caballero T, Crujeiras R, Soto-Varela A. Age-adjusted normality patterns for posturography by Sway Star system. Eur Arch Otorhinolaryngol. 2013;270(12):3169-3175. doi:10.1007/s00405-013-2508-y

15. Mancini M, King L, Salarian A, Holmstrom L, McNames J, Horak FB. Mobility Lab to assess balance and gait with synchronized body-worn sensors. J Bioeng Biomed Sci. 2011;1:007. doi:10.4172/ 2155-9538.S1-007

16. Mancini M, Horak FB. Potential of APDM Mobility Lab for the monitoring of the progression of Parkinson's disease. Expert Rev Med Devices. 2016;13(5):455-462. doi:10.1586/17434440.2016.1153421

17. Godinho C, Domingos J, Cunha G, et al. A systematic review of the characteristics and validity of monitoring technologies to assess Parkinson's disease. J Neuroeng Rehabil. 2016;13:24. doi:10.1186/ s12984-016-0136-7 
18. Sankarpandi SK, Baldwin AJ, Ray J, Mazzà C. Reliability of inertial sensors in the assessment of patients with vestibular disorders: a feasibility study. BMC Ear Nose Throat Disorders. 2017;17:1. doi:10.1186/s12901-017-0034-z

19. Lanovaz JL, Oates AR, Treen TT, Unger J, Musselman KE. Validation of a commercial inertial sensor system for spatiotemporal gait measurements in children. Gait Posture. 2017;51:14-19. doi:10.1016/j.gaitpost.2016.09.021

20. Buchman AS, Leurgans SE, Weiss A, et al. Associations between quantitative mobility measures derived from components of conventional mobility testing and Parkinsonian gait in older adults. PLoS One. 2014;9(1):e86262. doi:10.1371/journal.pone.0086262

21. Lindemann U, Nilssen RM, Nicolai SE, Becker C, Chiari L. Assessment of balance in unsupported standing with elderly inpatients by force plate and accelerometers. Aging Clin Exp Res. 2012;24 (1):37-41. doi:10.1007/BF03325352

22. Iluz T, Gazit E, Herman T, et al. Automated detection of missteps during community ambulation in patients with Parkinson's disease: a new approach for quantifying fall risk in the community setting. J Neuroeng Rehabil. 2014;11:48. doi:10.1186/1743-0003-11-48

23. Xsens Inc. MVN user manual, revision $Z$ [homepage on the Internet]. 2020. Available from: https://www.xsens.com/hubfs/Downloads/user manual/MVN_User_Manual.pdf. Accessed September 08, 2020.

24. Spain RI, George RJ, Salarian A, et al. Body-worn motion sensors detect balance and gait deficits in people with multiple sclerosis who have normal walking speed. Gait Posture. 2012;35(4):573-578. doi:10.1016/j.gaitpost.2011.11.026

25. Wüest S, Massé F, Aminian K, Gonzenbach R, de Bruin ED. Reliability and validity of the inertial sensor-based Timed "Up and Go" test in individuals affected by stroke. J Rehabil Res Dev. 2016;53 (5):599-610. doi:10.1682/JRRD.2015.04.0065

26. Nyan MN, Tay FEH, Murugasu E. A wearable system for pre-impact fall detection. $J$ Biomech. 2008;41(16):3475-3481. doi:10.1016/j. jbiomech.2008.08.009

27. Najafi B, Horn D, Marclay S, Crews RT, Wu S, Wrobel JS. Assessing postural control and postural control strategy in diabetes patients using innovative and wearable technology. J Diabetes Sci Technol. 2010;4(4):780-791. doi:10.1177/193229681000400403

28. Bonora G, Carpinella I, Cattaneo D, Chiari L, Ferrarin M. A new instrumented method for the evaluation of gait initiation and step climbing based on inertial sensors: a pilot application in Parkinson's disease. J Neuroeng Rehabil. 2015;12:45. doi:10.1186/s12984-015-0038-0

29. Xu J, Bao T, Lee UH, et al. Configurable, wearable sensing and vibrotactile feedback system for real-time postural balance and gait training: proof-of-concept. J Neuroeng Rehabil. 2017;14(1):102. doi:10.1186/s12984-017-0313-3

30. Kotas R, Janc M, Kamiński M, Marciniak P, Zamysłowska-Szmytke E, Tylman W. Evaluation of agreement between static posturography methods employing tensometers and inertial sensors. IEEEC Access. 2019;7:164120-164126. doi:10.1109/ACCESS.2019.2952496

31. Valldeperes A, Altuna X, Martinez-Basterra Z, et al. Wireless inertial measurement unit (IMU)-based posturography. Eur Arch Otorhinolaryngol. 2019;276(11):3057-3065. doi:10.1007/s00405019-05607-1

32. Kingma H, Felipe L, Gerards MC, et al. Vibrotactile feedback improves balance and mobility in patients with severe bilateral vestibular loss. J Neurol. 2019;266(Suppl 1):19-26. doi:10.1007/s00415018-9133-z

33. Alessandrini M, Micarelli A, Viziano A, et al. Body-worn triaxial accelerometer coherence and reliability related to static posturography in unilateral vestibular failure. Acta Otorhinolaryngol Ital. 2017;37(3):231-236. doi:10.14639/0392-100X-1334

34. Ma C, Wong DW, Lam WK, Wan AH, Lee WC. Balance Improvement effects of biofeedback systems with state-of-the-art wearable sensors: a systematic review. Sensors (Basel). 2016;16 (4):434. doi:10.3390/s16040434
35. Horak FB, King L, Mancini M. Role of body-worn movement monitor technology for balance and gait rehabilitation. Phys Ther. 2015;95 (3):461-470. doi:10.2522/ptj.20140253

36. Patel S, Park H, Bonato P, Chan L, Rodgers M. A review of wearable sensors and systems with application in rehabilitation. $J$ Neuroeng Rehabil. 2012;9:21. doi:10.1186/1743-0003-9-21

37. Hubble RP, Naughton GA, Silburn PA, Cole MH. Wearable sensor use for assessing standing balance and walking stability in people with Parkinson's disease: a systematic review. PLoS One. 2015;10(4): e0123705. doi:10.1371/journal.pone.0123705

38. Rucco R, Sorriso A, Liparoti M, et al. Type and location of wearable sensors for monitoring falls during static and dynamic tasks in healthy elderly: a review. Sensors (Basel). 2018;18(5):1613. doi: $10.3390 / \mathrm{s} 18051613$

39. Howcroft J, Kofman J, Lemaire ED. Review of fall risk assessment in geriatric populations using inertial sensors. J Neuroeng Rehabil. 2013;10(1):91. doi:10.1186/1743-0003-10-91

40. Özdemir AT. An analysis on sensor locations of the human body for wearable fall detection devices: principles and practice. Sensors (Basel). 2016;16(8):1161. doi:10.3390/s16081161

41. Pinho AS, Salazar AP, Hennig EM, Spessato BC, Domingo A, Pagnussat AS. Can we rely on mobile devices and other gadgets to assess the postural balance of healthy individuals? A systematic review. Sensors (Basel). 2019;19(13):E2972. doi:10.3390/s19132972

42. Howcroft J, Lemaire ED, Kofman J. Wearable-sensor-based classification models of faller status in older adults. PLoS One. 2016;11(4): e0153240. doi:10.1371/journal.pone. 0153240

43. Mancini M, Salarian A, Carlson-Kuhta P, et al. ISway: a sensitive, valid and reliable measure of postural control. $J$ Neuroeng Rehabil. 2012;9:59. doi:10.1186/1743-0003-9-59

44. Brown HJ, Siegmund GP, Guskiewicz KM, van den Doel K, Cretu E, Blouin J-S. Development and validation of an objective balance error scoring system. Med Sci Sports Exerc. 2014;46(8):1610-1616. doi:10.1249/MSS.0000000000000263

45. King LA, Horak FB, Mancini M, et al. Instrumenting the balance error scoring system for use with patients reporting persistent balance problems after mild traumatic brain injury. Arch Phys Med Rehabilit. 2014;95(2):353-359. doi:10.1016/j.apmr.2013.10.015

46. Whitney SL, Roche JL, Marchetti GF, et al. A comparison of accelerometry and center of pressure measures during computerized dynamic posturography: a measure of balance. Gait Posture. 2011;33(4):594-599. doi:10.1016/j.gaitpost.2011.01.015

47. Salarian A, Horak FB, Zampieri C, Carlson-Kuhta P, Nutt JG, Aminian K. iTUG, a sensitive and reliable measure of mobility. IEEE Trans Neural Syst Rehabil Eng. 2010;18(3):303-310. doi:10.1109/TNSRE.2010.2047606

48. Zampieri C, Salarian A, Carlson-Kuht P, Aminian K, Nutt JG, Horak FB. The instrumented timed up and go test: potential outcome measure for disease modifying therapies in Parkinson's disease. J Neurol Neurosurg Psychiatry. 2010;81(2):171-176. doi:10.1136/ jnnp.2009.173740

49. Gerhardy T, Gordt K, Jansen CP, Schwenk M. Towards using the instrumented Timed Up and-Go Test for screening of sensory system performance for balance control in older adults. Sensors (Basel). 2019;19(3):622. doi:10.3390/s19030622

50. O'Sullivan M, Blake C, Cunningham C, Boyle G, Finucane C. Correlation of accelerometry with clinical balance tests in older fallers and non-fallers. Age Ageing. 2009;38(3):308-313. doi:10.1093/ ageing/afp009

51. Mellone S, Tacconi C, Schwickert L, Klenk J, Becker C, Chiari L. Smartphone-based solutions for fall detection and prevention: the FARSEEING approach. Z Gerontol Geriatr. 2012;45(8):722-727. doi:10.1007/s00391-012-0404-5

52. Rey-Martinez J. RombergLab 4+, Wireless IMU Posturography. Available from: https://apps.apple.com/us/app/romberglab/ id1440287860. Accessed August 17, 2020. 
53. Lee BC, Kim J, Chen S, Sienko KH. Cell phone based balance trainer. J Neuroeng Rehabil. 2012;9:10. doi:10.1186/1743-0003-9-10

54. Fleury A, Mourcou Q, Franco C, Diot B, Demongeot J, Vuillerme N Evaluation of a smartphone-based audio-biofeedback system for improving balance in older adults - a pilot study. Proceedings of the 2013 35th Annual International Conference of the IEEE Engineering in Medicine and Biology Society (EMBC); July 3-7, 2013; Osaka, Japan. 1198-1201.

55. Sposaro F, Tyson G. iFall: an Android application for fall monitoring and response. Conf Proc IEEE Eng Med Biol Soc. 2009;2009:6119-6122. doi:10.1109/IEMBS.2009.5334912

56. Cohen HS, Mulavara AP, Peters BT, Sangi-Haghpeykar H, Bloomberg JJ. Standing balance tests for screening people with vestibular impairments. Laryngoscope. 2014;124(2):545-550. doi:10.1002/lary.24314

57. Teixeira P, Nunes F, Silva PA, Teixeira L Mover activity monitor and fall detector for Android. Proceedings of the Mobile Wellness Workshop at Mobile HCI 2011 (MW2011); Aug 30-Sep 2, 2011; Stockholm, Sweden.

58. Soto-Varela A, Gayoso-Diz P, Faraldo-García A, et al. Optimising costs in reducing rate of falls in older people with the improvement of balance by means of vestibular rehabilitation (ReFOVeRe study): a randomized controlled trial comparing computerised dynamic posturography vs mobile vibrotactile posturography system. BMC Geriatr. 2019;19(1):1. doi:10.1186/s12877-018-1019-5

59. Faraldo-Garcia A, Sandos-Perez S, Crujeiras R, Labella-Caballero T, Soto-Varela A. Comparative study of computerized dynamic posturography and the Sway Star system in healthy subjects. Acta Otolaryngol. 2012;132(3):271-276. doi:10.3109/ 00016489.2011.637177

60. de Bruin ED, Hartmann A, Uebelhart D, Murer K, Zijlstra W. Wearable systems for monitoring mobility-related activities in older people: a systematic review. Clin Rehabil. 2008;22(10-11):878-895. doi:10.1177/0269215508090675

61. Chaudhuri S, Thompson H, Demiris G. Fall detection devices and their use with older adults: a systematic review. J Geriat Phys Ther. 2014;37(4):178-196. doi:10.1519/JPT.0b013e3182abe779

62. Horak FB, Mancini M. Objective biomarkers of balance and gait for Parkinson's disease using body-worn sensors. Mov Disord. 2013;28 (11):1544-1551. doi:10.1002/mds.25684

63. Sparaco M, Lavorgna Conforti R, Tedeschi G, Bonavita S. The role of wearable devices in multiple sclerosis. Mult Scler Int. 2018;2018:7627643. doi:10.1155/2018/7627643
64. Weiss A, Herman T, Giladi N, Hausdorff JM. Objective assessment of fall risk in Parkinson's disease using a body-fixed sensor worn for 3 days. PLoS One. 2014;9(5):e96675. doi:10.1371/journal. pone.0096675

65. Herman T, Weiss A, Brozgol M, Giladi N, Hausdorff JM. Gait and balance in Parkinson's disease subtypes: objective measures and classification considerations. J Neurol. 2014;261(12):2401-2410. doi:10.1007/s00415-014-7513-6

66. Mirelman A, Heman T, Yasinovsky K, et al. Fall risk and gait in Parkinson's disease: the role of the LRRK2 G2019S mutation. Mov Disord. 2013;28(12):1683-1690. doi:10.1002/mds.25587

67. Rovini E, Maremmani C, Cavallo F. How wearable sensors can support Parkinson's disease diagnosis and treatment: a systematic review. Front Neurosci. 2017;11:555. doi:10.3389/fnins.2017.00555

68. Hasegawa N, Shah VV, Carlson-Kuhta P, Nutt JG, Horak FB, Mancini M. How to select balance measures sensitive to Parkinson's disease from body-worn inertial sensors separating the trees from the forest. Sensors (Basel). 2019;19(15):3320. doi:10.3390/s19153320

69. Brugnera C, Bittar RSM, Greters ME, Basta D. Effects of vibrotactile vestibular substitution on vestibular rehabilitation - preliminary study. Braz J Otorhinolaryngol. 2015;81(6):616-621. doi:10.1016/j. bjorl.2015.08.013

70. Drover D, Howcroft J, Kofman J, Lemaire ED. Faller classification in older adults using wearable sensors based on turn and straight-walking accelerometer-based features. Sensors (Basel). 2017;17(6):1321. doi:10.3390/s17061321

71. Schwickert L, Becker C, Lindemann U, et al; for the FARSEEING Consortium and the FARSEEING Meta Database Consensus Group. Fall detection with body-worn sensors: a systematic review. Z Gerontol Geriatr. 2013;46(8):706-719. doi:10.1007/s00391-013$0559-8$

72. Deveze A, Bernard-Demanze L, Xavier F, Lavieille J-P, Elziere M. Vestibular compensation and vestibular rehabilitation. Current concepts and new trends. Neurophysiol Clin. 2014;44(1):49-57. doi:10.1016/j.neucli.2013.10.138

73. Borel L, Alescio-Lautier B. Posture and cognition in the elderly: interaction and contribution to the rehabilitation strategies. Neurophysiol Clin. 2014;44(1):95-107. doi:10.1016/j.neucli.201 3.10 .129
Clinical Interventions in Aging

\section{Publish your work in this journal}

Clinical Interventions in Aging is an international, peer-reviewed journal focusing on evidence-based reports on the value or lack thereof of treatments intended to prevent or delay the onset of maladaptive correlates of aging in human beings. This journal is indexed on PubMed Central, MedLine, CAS, Scopus and the Elsevier

\section{Dovepress}

Bibliographic databases. The manuscript management system is completely online and includes a very quick and fair peer-review system, which is all easy to use. Visit http://www.dovepress.com/ testimonials.php to read real quotes from published authors. 